\title{
Investigation of absorption of laser radiation by copper- and nickel-based powders to obtain gradient materials by selective laser melting
}

\author{
Anna Voznesenskaya, Dmitriy Kochuev, Artem Raznoschikov, Andrey Kireev, and \\ Kirill Khorkov \\ Vladimir State University, 600000 Vladimir, Russia
}

\begin{abstract}
The absorption of laser radiation of powder materials based on copper and nickel is studied. The dependence of the temperature of the powder material on the power of the incident laser radiation is obtained. A comparison of the values obtained by different measurement methods is presented.
\end{abstract}

With selective laser melting (SLM), the properties of the deposited layer, such as uniformity, density, contact with the surface of the part, depend on a number of technological parameters: power density in the laser spot, scanning speed over the surface, nature of the filing of the deposited material, fractional and chemical composition, as well as the absorption capacity of the deposited material [1]. Studies of the absorption of powder materials are of particular interest for the development of selective laser melting, because it allows you to determine the processing parameters suitable for defect-free production of the part. A preliminary knowledge of such information is important for obtaining a more uniform and reproducible laser melting process [2]. The absorption capacity (A) of a material is defined as the ratio of absorbed radiation to incident radiation. Typically, reflection coefficient $(\mathrm{R})$ is measured in experiments. Absorption capacity is calculated by the formula: $A=1 \pm R$ [1]. Since powder materials are a porous structure, part of the incident radiation is absorbed by the outer surface of the particles. Another part of the radiation penetrates deep into the powder through the pores, interacting with the underlying particles [3]. The absorption of pores by radiation approaches the absorption of a black body. The surface structure of the powder layer changes during laser melting and this provokes a change in absorbency.

Figure 1 shows a diagram of an experiment to study the absorption efficiency of laser radiation: 1 - Laser system; 2 - Beam Expander; 3 - Laser beam; 4 - Powder material; 5 Thermocouple; 6 - Analog digital converter; 7 - PC; 8 - Photopyrometer.

A fiber laser with a wavelength of $1060 \mathrm{~nm}$ was used as a radiation source. The defocused laser beam was directed onto the surface of the powder material; the diameter of the laser beam on the surface of the powder material was about $8 \mathrm{~mm}$. Thermal sensors were connected to an analog-to-digital converter. The temperature was measured using a photopyrometer.

Before starting measurements, it is necessary to achieve thermal equilibrium [4]. The range of variation of the laser radiation power was chosen in such a way as to exclude melting, evaporation, and other changes in the state of aggregation of the powders. 


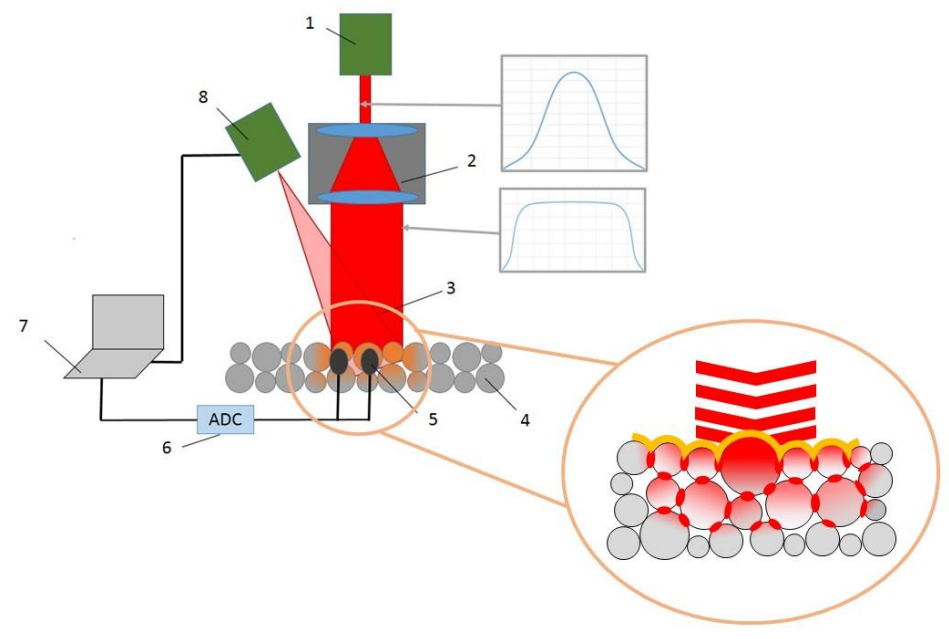

Fig. 1. Scheme for measuring the absorption of powder materials.

Then, by the method of selective laser melting, 2 types of heterogeneous powder material of a chromium-nickel alloy and copper were deposited in order to obtain a gradient material. The shape and fractional composition of powder materials were studied by scanning electron microscopy. Using a diffractometer, the correlation of the elements inside the deposited layer was revealed.

\section{References}

1. N.B. Delone, Moskow: Science 280 (1989)

2. S. Alexandrov et al., Journal of Physics: Conference Series. - IOP Publishing, 1164 (1) 012001 (2019)

3. I. Yu. Smurov et al., Vestnik MGTU STANKIN 2 (4) 144-146 (2011)

4. A. S. Chernikov et al. Journal of Physics: Conference Series. IOP Publishing, 1109 (1) 012062 (2018) 